\title{
ARTICLE
}

Epidemiology

\section{A prediction model for underestimation of invasive breast cancer after a biopsy diagnosis of ductal carcinoma in situ: based on 2892 biopsies and 589 invasive cancers}

Claudia J. C. Meurs ${ }^{1}$, Joost van Rosmalen ${ }^{2}$, Marian B. E. Menke-Pluijmers ${ }^{3}$, Bert P. M. ter Braak ${ }^{4}$, Linda de Munck ${ }^{5}$, Sabine Siesling ${ }^{5}$ and $^{6}$ Pieter J. Westenend ${ }^{6,7}$

BACKGROUND: Patients with a biopsy diagnosis of ductal carcinoma in situ (DCIS) might be diagnosed with invasive breast cancer at excision, a phenomenon known as underestimation. Patients with DCIS are treated based on the risk of underestimation or progression to invasive cancer. The aim of our study was to expand the knowledge on underestimation and to develop a prediction model.

METHODS: Population-based data were retrieved from the Dutch Pathology Registry and the Netherlands Cancer Registry for DCIS between January 2011 and June 2012.

RESULTS: Of 2892 DCIS biopsies, $21 \%$ were underestimated invasive breast cancers. In multivariable analysis, risk factors were highgrade DCIS (odds ratio (OR) 1.43, 95\% confidence interval (CI): 1.05-1.95), a palpable tumour (OR 2.22, 95\% Cl: 1.76-2.81), a BI-RADS (Breast Imaging Reporting and Data System) score 5 (OR 2.36, 95\% Cl: 1.80-3.09) and a suspected invasive component at biopsy (OR $3.84,95 \% \mathrm{Cl}: 2.69-5.46)$. The predicted risk for underestimation ranged from 9.5 to $80.2 \%$, with a median of $14.7 \%$. Of the 596 invasive cancers, $39 \%$ had unfavourable features.

CONCLUSIONS: The risk for an underestimated diagnosis of invasive breast cancer after a biopsy diagnosis of DCIS is considerable. With our prediction model, the individual risk of underestimation can be calculated based on routinely available preoperatively known risk factors (https://www.evidencio.com/models/show/1074).

British Journal of Cancer (2018) 119:1155-1162; https://doi.org/10.1038/s41416-018-0276-6

\section{BACKGROUND}

Patients with ductal carcinoma in situ (DCIS) are treated based on the risk of underestimation or progression to invasive cancer. The standard treatment for patients with a biopsy diagnosis of DCIS is wide local excision with radiation or mastectomy. Often a sentinel lymph node (SLN) biopsy is advised for axillary staging. ${ }^{1,2}$ Both the standard treatment and the use of the SLN biopsy can constitute overtreatment. The standard treatment might be disproportionate for screen-detected DCIS patients who have a high chance that the DCIS would not even have been detected during their lifetime. $^{3}$ It has been estimated that between 14 and $53 \%$ of DCIS progress into invasive breast cancer. 4,5

To address overtreatment, phase III trials investigate the safety of active surveillance of DCIS patients at low risk for developing or having invasive breast cancer. ${ }^{6-11}$ Active surveillance is based on the result of the biopsy. By modelling of active surveillance of DCIS patients, the disease-specific cumulative mortality was related to underestimation. ${ }^{12,13}$ Underestimation is the phenomenon that the invasive breast cancer is undetected at preoperative biopsy and only becomes evident after pathological examination of the excision material. The use of the SLN biopsy can also constitute overtreatment. The SLN biopsy is done if a mastectomy is chosen, and also for patients undergoing wide local excision who are at high risk of having an underestimated invasive breast cancer. ${ }^{1,2}$ The reported risk of underestimation varies from 14 to $43 \%{ }^{14,15}$, and in a meta-analysis it was estimated to be $25.9 \%(95 \%$ confidence interval $(\mathrm{Cl}): 22.5-29.5)^{16}$. These rates indicate that many patients will still have the diagnosis of DCIS after examination of the excision material, and thus the SLN biopsy would not have been necessary.

Knowledge on the risk of underestimation is important in selecting high-risk or low-risk patients for treatment or active surveillance. The most frequently reported risk factors for underestimation are DCIS grade and factors found with radiological diagnostic work-up, such as the size of the lesion, mass on mammography or ultrasonography, and the Breast Imaging Reporting and Data System (BI-RADS) score. ${ }^{14-27}$ Furthermore, these studies reported that the risk of underestimation was

\footnotetext{
${ }^{1}$ CMAnalyzing, Gounodstraat 16, 6904 HC Zevenaar, The Netherlands; ${ }^{2}$ Department of Biostatistics, Erasmus MC, University Medical Center Rotterdam, Wytemaweg 80,3015 CN Rotterdam, The Netherlands; ${ }^{3}$ Department of Surgery, Albert Schweitzer Hospital, PO Box 444, 3300 AK Dordrecht, The Netherlands; ${ }^{4}$ Department of Radiology, Albert Schweitzer Hospital, PO Box 444, 3300 AK Dordrecht, The Netherlands; ${ }^{5}$ Department of Research, Netherlands Comprehensive Cancer Organisation, PO Box 19079 , 3501 DB Utrecht, The

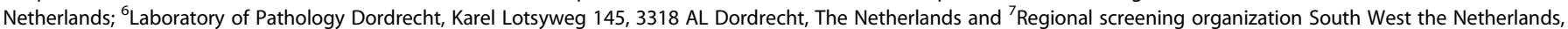
Maasstadweg 12, 3079 DZ Rotterdam, The Netherlands

Correspondence: Pieter J. Westenend (pwestenend@paldordrecht.nl)
}

Received: 6 April 2018 Revised: 4 September 2018 Accepted: 6 September 2018

Published online: 17 October 2018 
A prediction model for underestimation of invasive breast cancer after a...

CJC Meurs et al.

Table 1. Results of previous studies on risk factors for underestimation

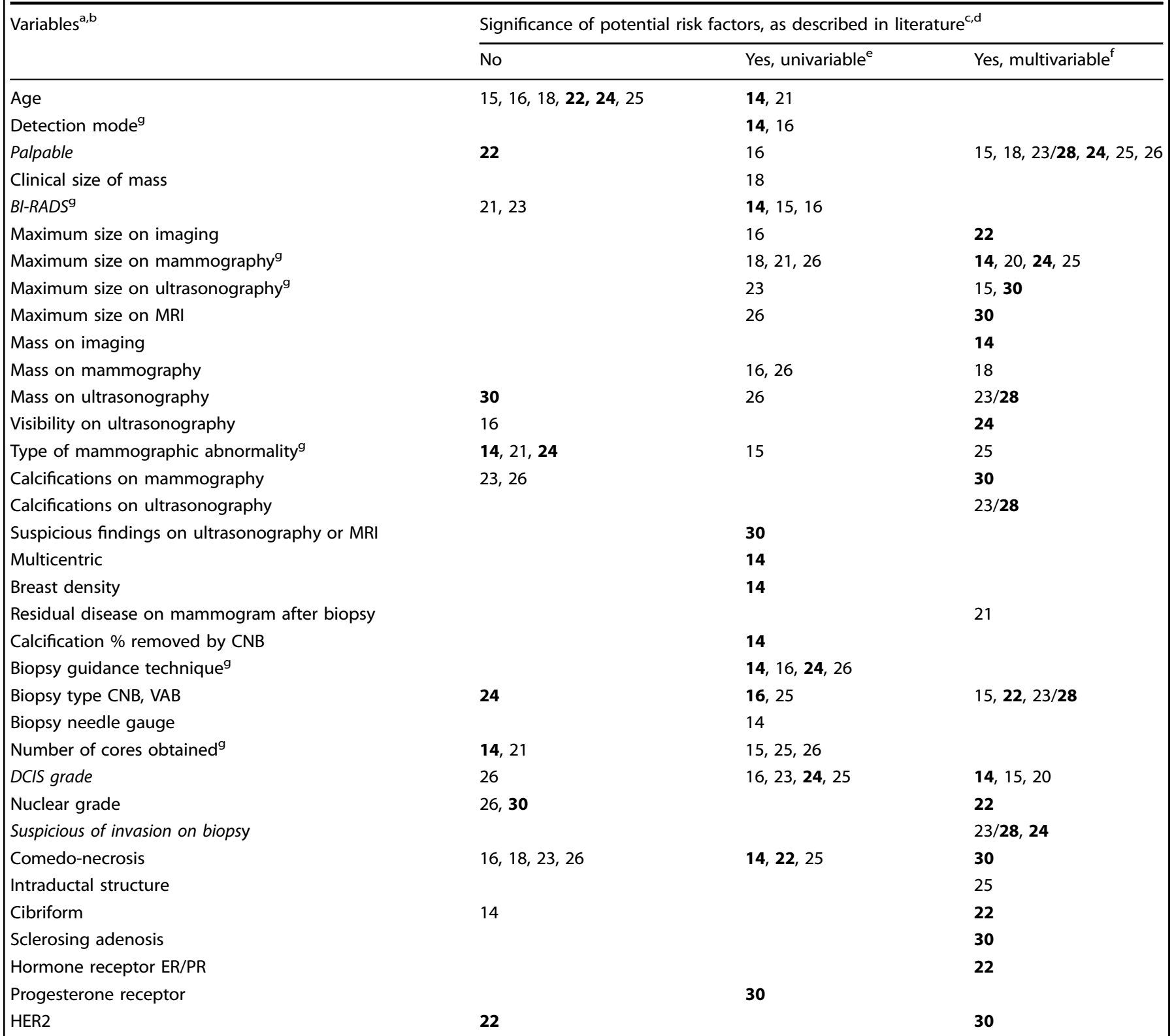

${ }^{a}$ Variables in bold are variables that were analysed in this study

bVariables that were analysed but were not statistically significant in any study were: mass on MRI (30), mass on ultrasonography or MRI (30), abnormality on mammography; mass, asymmetry or distortion (23), calcifications on imaging (22), suspicious findings on ultrasonography (30), suspicious findings on MRI $(30)$, solid $(14,22)$ papillary $(14,22)$, micropapillary $(14,22)$, necrosis $(22)$, oestrogen receptor $(14,30)$, period from breast biopsy to surgery $(25)$, Van Nuys grouping (23), family history (21), menopausal status (21) and type of first resection (18)

CListed are 12 studies with at least 100 cases of underestimation

${ }^{d}$ References in bold are of the five studies that developed a prediction model

Reference ${ }^{16}$ presents results of random-effect logistic regression models in a meta-analysis

${ }^{\mathrm{T}}$ The multivariable significant variables of ref. ${ }^{23}$ were used in a prediction model, as described in ref. ${ }^{28}$

${ }^{9}$ The categories of these variables were not uniformly defined between studies

associated with age, palpability, histologic suspicion of invasion, image guidance method, biopsy device and other factors. An overview of the found risk factors for underestimation is given in Table 1. Based on risk factors, several studies developed prediction models with the purpose to select patients for SLN biopsy ${ }^{14,17,24,28-30}$.

Besides the underestimation rate, other factors are useful for making a treatment plan for a patient diagnosed at biopsy with DCIS. First of all, for some of these patients, no residual disease is found in the excision material; this is defined as minimal-volume DCIS. A rate of $9.3 \%$ was reported. ${ }^{31}$ Second, of the underestimated invasive breast cancers the information on unfavourable features is of interest; the reported Her2Neu status is quite high $^{22,23}$ and the hormonal receptor statuses vary ${ }^{21-23,25,26}$.

The diversity of identified risk factors for underestimation has resulted in differences between the clinical guidelines used in different countries. For example, according to the NICE guideline (United Kingdom) for the use of the SLN biopsy, risk factors for 
underestimation are a palpable mass or extensive micro-calcifications, while according to the Dutch guideline, these are age $<55$ years, intermediate-grade or high-grade DCIS, a mass on mammography and a suspected invasive component based on biopsy. For active surveillance, the main criterion for patient selection in low-risk DCIS trials are DCIS grade, and patients with mass or other relevant factors are excluded.

The diversity in risk factors might be due to the study designs, since the investigated potential risk factors varied and many studies on underestimation were single institution studies with limited number of cases. Information at the population level is lacking. In addition, there is hardly any data on minimal-volume DCIS nor on the presence of unfavourable features of the underestimated invasive breast cancer.

The aim of our study was to expand the knowledge on underestimation of invasive breast cancer for patients with a biopsy diagnosis of DCIS in routine clinical practice in the Netherlands and to develop a prediction model based on population data. We also analysed the association of predicted risk with minimal-volume DCIS and with the occurrence of unfavourable features of the underestimated invasive breast cancer. The results could contribute to a treatment plan that is both patient-specific and helps in reducing overtreatment.

\section{METHODS}

Study design and population

This study used retrospective data that were nationwide. Data were received from the Dutch Pathology Registry, which is managed by PALGA (the nationwide network and registry of histopathology and cytopathology in the Netherlands) and were matched with data from the Netherlands Cancer Registry (NCR), which is hosted by the IKNL (the Netherlands Comprehensive Cancer Organization). The Dutch Pathology Registry contains all the reports written by pathologists of material examined in all Dutch Pathology Laboratories. ${ }^{32}$ The NCR contains information that is collected and coded by specially trained registration clerks from the hospitals' patient files of every patient with cancer, after notification from PALGA. ${ }^{33}$

Lesions were selected from PALGA, since this study is based on the biopsy diagnosis and the NCR registers the final diagnosis at excision. Histological breast biopsies were selected that were performed in the period 1 January 2011 until 30 June 2012. The diagnosis should be carcinoma in situ, with no invasive cancer in the same biopsy, no lymph node metastases found preoperative and also no melanoma in situ, Morbus Paget or Morbus Bowen. DCIS with micro-invasion was not included, nor were intracystic carcinoma, lobular carcinoma in situ and ductal hyperplasia lesions. Based on the PALGA conclusion (free text field) information on the diagnosis, DCIS grade, suspected invasive component, synchronous contralateral tumour and ipsilateral history were coded. The data were extended with those registered by the NCR: age, ipsilateral history, detection mode, palpability, BI-RADS score, preoperative magnetic resonance imaging (MRI), multidisciplinary team meeting, type of first resection, nodal status, and of the invasive cancers, the morphology, grade, the receptors ER, PR, Her2Neu and tumour size. Lesions were excluded in case of incomplete registration, primarily no excision of the lesion, a biopsy diagnosis that was inconclusive as to whether the lesion was benign or DCIS and in case of an ipsilateral history of DCIS or invasive breast cancer.

Data were categorized as follows: the category detection mode consisted of screen-detected DCIS (DCIS detected within 12 months after a positive mammography at the populationbased screening programme) and otherwise detected DCIS (all other DCIS). DCIS grade was categorized into low, intermediate or high. If the tumour consisted of two different grades or if the grade was inconclusive, the highest DCIS grade was chosen.
Suspected invasive component was coded 'yes' if it was mentioned as such in the conclusion of the pathology report and if it was not refuted with potential additional staining. For the BI-RADS score, no subgroup information for score 4 was available. ${ }^{34}$ A synchronous contralateral lesion was defined as DCIS or invasive breast cancer in both breasts with a difference in incidence date of $<3$ months. Underestimation was defined as invasive cancer or micro-invasion found at excision after a biopsy diagnosis DCIS. Tumours were graded according to the Bloom-Richardson grade or another equivalent method. Tumour size and nodal status were used to categorize the tumor node metastasis (TNM) stage. ${ }^{35}$ Underestimated invasive breast cancers were categorized based on unfavourable features. In the Dutch guideline ${ }^{36}$, they were defined as features that, if present, would mean that systemic therapy would be recommended, because the absolute 10 -year mortality risk was at least $15 \%$. These features of the invasive cancers were:

- Her2Neu positive with size $>5 \mathrm{~mm}$.

- Age $<35$ years, except size $\leq 10 \mathrm{~mm}$ with grade I.

- Size $>10 \mathrm{~mm}$ but $\leq 20 \mathrm{~mm}$ with grade II or III.

- Size $>20 \mathrm{~mm}$.

- Positive lymph nodes.

Statistical analysis

The data were analysed to investigate associations and to develop a prediction model. First, the distribution of patient characteristics and potential risk factors was compared between patients with and without underestimated invasive breast cancer for the nonmissing values, using the Mann-Whitney test or the Pearson's $X^{2}$ test. The associations between potential risk factors were analysed with the Pearson's $X^{2}$ test or the Fisher's exact test. The risk for underestimation of invasive breast cancer was analysed with logistic regression analysis. The threshold for significance of risk factors was the two-sided $p$ value of 0.05 . In this logistic regression, we only included characteristics that were known as independent variables prior to operation: age, detection mode, palpability, BI-RADS score, DCIS grade and suspected invasive component at biopsy. The decision to do a preoperative MRI and the type of first resection were not included in the model, because no causal association with underestimation was expected. Next, to ensure that all relevant variables were included in the prediction model, the independent variables were chosen via stepwise backward selection with a $p$ value threshold for elimination of $p<$ 0.20. In the prediction model, age was tested multiple times: continuously using both linear and quadratic terms and dichotomously with thresholds of 40,45 and 55 years for comparison with previous publications.,6,21 Interaction was tested for combinations that were clinically the most plausible: suspected invasive component and DCIS grade, age $<45$ years (based on cutoff age in active surveillance) in combination with BI-RADS score, or palpability, or DCIS grade. To account for missing data, multiple imputation with fully conditional specification was used in the multivariable logistic analysis. Twenty imputed data sets were generated, and the results were pooled according to Rubin's rules. Based on the imputed data, a formula was defined to predict the risk. Finally, internal validation of the model was performed with bootstrap repetitions (200 times). The logistic regression model was evaluated with the area under the curve (AUC) of the receiver operating characteristic (ROC). Based on the predicted risks, patients were divided into five subgroups, and the association with minimal-volume DCIS and unfavourable features was analysed with the $p$-trend test for proportions. The analyses were done with STATA statistics/data analysis, version 13.1, StataCorp, Texas and in $\mathrm{R}$, with the rms package for the evaluation of the predictive performance and the mice package for multiple imputation. 


\section{RESULTS}

Of 3281 lesions that were selected with a preoperative biopsy diagnosis DCIS, 64 (2.0\%) were excluded because they were not registered in the NCR, and $15(0.5 \%)$ because registration was incomplete. In addition, to answer the research question accurately, more were excluded: $60(1.8 \%)$ that did primarily not undergo excision, 143 (4.4\%) for which the biopsy diagnosis was inconclusive and 107 (3.3\%) with an ipsilateral history of DCIS or invasive breast cancer, resulting in 2892 DCIS diagnoses included in the study. Of these, $379(13 \%)$ had missing data for one or more potential risk factor: 148 for palpability, 223 for BI-RADS score, 84 for DCIS grade and 81 for detection mode.

Of the 2892 DCIS diagnoses at biopsy, 596 (20.6\%) were underestimated, as the diagnosis was invasive breast cancer at excision. Table 2 shows patient and biopsy characteristics and their relation with underestimation. Of biopsy DCIS, 66\% was screen detected, $22 \%$ was palpable, $13 \%$ had a BI-RADS score 3 , $75 \%$ had a BI-RADS score $4,12 \%$ had a BI-RADS score 5 and $5 \%$ had a suspected invasive component at biopsy. The DCIS grade distribution was $15 \%$ low, 39\% intermediate and $46 \%$ high ( $p=$ 0.001 ). Of the intermediates, $13 \%$ were low to intermediate or consisted of both low-grade and intermediate-grade DCIS, 21\% were intermediate to high grade or consisted of both intermediate-grade and high-grade DCIS. The underestimation rate was $21 \%$ on average for all cases, $26 \%$ for non-screendetected lesions, $36 \%$ for palpable lesions, $41 \%$ for BI-RADS score 5 and $23 \%$ for high-grade DCIS ( $p$ values between different categories were $<0.001$ for all variables). The risk factors with the greatest differences in underestimation rate for subgroups were palpability, with a $20 \%$ higher rate for palpable than for nonpalpable lesions, BI-RADS score, with a $25 \%$ higher rate for $\mathrm{BI}-$ RADS score 5 than for score 3 , and suspected invasive component, with a $31 \%$ higher rate for suspected invasive component than for none. Of 596 invasive breast cancers, 47 were $\mathrm{T} 1 \mathrm{mi}$ and 207 were T1a. The underestimation rate when filtering out all lesions of $5 \mathrm{~mm}$ or smaller was $11.8 \%(n=342)$.

Table 3 shows the results of univariable and multivariable analysis of the risk for underestimation of preoperatively known potential risk factors for invasive breast cancer. Age and detection mode were statistically significant in univariable analysis, but not in multivariable analysis. Both were associated with palpability and BI-RADS score, and age was also associated with DCIS grade (shown in supplement 1 , along with other associations). In multivariable analysis, grade, palpability, BI-RADS score and a suspected component were significant.

For each of the 2892 DCIS, the risk of an underestimated invasive breast cancer was calculated based on the prediction model with the following formula:

Predicted risk $=\left(\frac{1}{1+\mathrm{e}^{- \text {score }}}\right) \times 100 \%$,

with score $=-2.1131+0.1555 \times$ detection_mode_otherwise + $0.7985 \times$ palpable $-0.1464 \times$ BI-RADS_score_3 $+0.8589 \times \mathrm{BI}-$ RADS_score_5 $+0.3111 \times$ intermediate_DCIS_grade $+0.3571 \times$ high_DCIS_grade $+1.3445 \times$ suspected_invasive_component, where for all risk factors: $1=$ if condition applies, $0=$ otherwise.

For example, the predicted risk is calculated as follows for a screen-detected DCIS which is non-palpable, has a BI-RADS score 4 , an intermediate-grade and no suspected invasive component: score $=-2.1131+0.1555 \times 0+0.7985 \times 0-0.1464 \times 0+$

$0.8589 \times 0+0.3111 \times 1+0.3571 \times 0+1.3445 \times 0=-1.802$, and

Predicted risk $=\left(\frac{1}{1+\mathrm{e}^{-(-1.802)}}\right) \times 100 \%=14.2 \%$.

The risk for an individual patient can be calculated in a user-
Table 2. Distribution of underestimation rate

\begin{tabular}{|c|c|c|c|c|c|c|}
\hline & \multirow{3}{*}{$\begin{array}{l}\text { All } \\
N\end{array}$} & \multicolumn{4}{|c|}{$\begin{array}{l}\text { Underestimated invasive } \\
\text { breast cancer }\end{array}$} & \multirow[t]{3}{*}{$p$ value } \\
\hline & & \multicolumn{2}{|l|}{ No } & \multicolumn{2}{|l|}{ Yes } & \\
\hline & & $N$ & $\%$ & $N$ & $\%$ & \\
\hline Total & 2892 & 2296 & $79.4 \%$ & 596 & $20.6 \%$ & \\
\hline $\begin{array}{l}\text { Age (years), mean } \\
\text { (range) }\end{array}$ & $\begin{array}{l}58.7 \\
(24-91)\end{array}$ & $58.9(3$ & $(30-88)$ & 57.8 & (24-91) & 0.033 \\
\hline Age categories & & & & & & $<0.001$ \\
\hline$<45$ years & 207 & 142 & $69 \%$ & 65 & $31 \%$ & \\
\hline$\geq 45$ years & 2685 & 2154 & $80 \%$ & 531 & $20 \%$ & \\
\hline Detection mode & & & & & & $<0.001$ \\
\hline Screen detected & 1850 & 1521 & $82 \%$ & 329 & $18 \%$ & \\
\hline Otherwise & 961 & 714 & $74 \%$ & 247 & $26 \%$ & \\
\hline Missing & 81 & 61 & $75 \%$ & 20 & $25 \%$ & \\
\hline Palpable & & & & & & $<0.001$ \\
\hline No & 2147 & 1794 & $84 \%$ & 353 & $16 \%$ & \\
\hline Yes & 597 & 380 & $64 \%$ & 217 & $36 \%$ & \\
\hline Missing & 148 & 122 & $82 \%$ & 26 & $18 \%$ & \\
\hline BI-RADS score & & & & & & $<0.001$ \\
\hline 3 & 365 & 306 & $84 \%$ & 59 & $16 \%$ & \\
\hline 4 & 1996 & 1638 & $82 \%$ & 358 & $18 \%$ & \\
\hline 5 & 308 & 183 & $59 \%$ & 125 & $41 \%$ & \\
\hline Missing & 223 & 169 & $76 \%$ & 54 & $24 \%$ & \\
\hline $\begin{array}{l}\text { DCIS histological grade } \\
\text { at biopsy }\end{array}$ & & & & & & 0.001 \\
\hline Low & 422 & 360 & $85 \%$ & 62 & $15 \%$ & \\
\hline Intermediate & 1083 & 866 & $80 \%$ & 217 & $20 \%$ & \\
\hline High & 1303 & 1006 & $77 \%$ & 297 & $23 \%$ & \\
\hline Missing & 84 & 64 & $76 \%$ & 20 & $24 \%$ & \\
\hline $\begin{array}{l}\text { Suspected invasive } \\
\text { component at biopsy }\end{array}$ & & & & & & $<0.001$ \\
\hline No & 2743 & 2222 & $81 \%$ & 521 & $19 \%$ & \\
\hline Yes & 149 & 74 & $50 \%$ & 75 & $50 \%$ & \\
\hline $\begin{array}{l}\text { Synchronous } \\
\text { contralateral breast } \\
\text { tumour }\end{array}$ & & & & & & 0.181 \\
\hline No & 2796 & 2225 & $80 \%$ & 571 & $20 \%$ & \\
\hline Yes & 96 & 71 & $74 \%$ & 25 & $26 \%$ & \\
\hline Preoperative MRI & & & & & & $<0.001$ \\
\hline No (or unknown) & 2188 & 1773 & $81 \%$ & 415 & $19 \%$ & \\
\hline Yes & 704 & 523 & $74 \%$ & 181 & $26 \%$ & \\
\hline $\begin{array}{l}\text { Preoperative } \\
\text { multidisciplinary team } \\
\text { meeting }\end{array}$ & & & & & & 0.364 \\
\hline No (or unknown) & 301 & 245 & $81 \%$ & 56 & $19 \%$ & \\
\hline Yes & 2591 & 2051 & $79 \%$ & 540 & $21 \%$ & \\
\hline First resection & & & & & & $<0.001$ \\
\hline Wide local excision & 1822 & 1510 & $83 \%$ & 312 & $17 \%$ & \\
\hline Mastectomy & 1070 & 786 & $73 \%$ & 284 & $27 \%$ & \\
\hline
\end{tabular}

friendly way with a calculation tool in Evidencio, https://www. evidencio.com/models/show/1074.

The predicted risks ranged from 9.5 to $80.2 \%$, the mean was $20.6 \%$ and the median was $14.7 \%$. The predicted risk for 
Table 3. Risk factors for underestimation

\begin{tabular}{|c|c|c|c|c|c|c|}
\hline \multirow{3}{*}{$\begin{array}{l}\text { Preoperative } \\
\text { patient and } \\
\text { lesion } \\
\text { characteristics }^{\mathrm{a}}\end{array}$} & \multicolumn{6}{|c|}{$\begin{array}{l}\text { Logistic regression analysis for underestimation of } \\
\text { invasive breast cancer }\end{array}$} \\
\hline & \multicolumn{3}{|c|}{ Univariable } & \multicolumn{3}{|c|}{ Multivariable $^{\mathrm{b}}$} \\
\hline & OR & $95 \% \mathrm{Cl}$ & $p$ value & OR & $95 \% \mathrm{Cl}$ & $p$ value \\
\hline Age (years) & & & & & & $\begin{array}{l}\text { not in } \\
\text { the } \\
\text { model }\end{array}$ \\
\hline$<45$ & 1.86 & $1.34-2.53$ & $<0.001$ & & & \\
\hline$\geq 45$ & 1 & & & & & \\
\hline \multicolumn{7}{|l|}{ Detection mode } \\
\hline Screen detected & 1 & & & 1 & & \\
\hline Otherwise & 1.60 & $1.33-1.93$ & $<0.001$ & 1.16 & $0.94-1.45$ & 0.164 \\
\hline \multicolumn{7}{|l|}{ Palpable } \\
\hline No & 1 & & & 1 & & \\
\hline Yes & 2.90 & $2.37-3.55$ & $<0.001$ & 2.22 & $1.76-2.81$ & $<0.001$ \\
\hline BI-RADS score & & & $<0.001$ & & & $<0.001$ \\
\hline 3 & 0.88 & $0.65-1.19$ & 0.487 & 0.86 & $0.64-1.17$ & 0.348 \\
\hline 4 & 1 & & & 1 & & \\
\hline 5 & 3.13 & $2.43-4.03$ & $<0.001$ & 2.36 & $1.80-3.09$ & $<0.001$ \\
\hline $\begin{array}{l}\text { DCIS histological } \\
\text { grade at biopsy }\end{array}$ & & & 0.001 & & & 0.078 \\
\hline Low & 1 & & & 1 & & \\
\hline Intermediate & 1.45 & $1.06-1.98$ & 0.017 & 1.36 & $0.99-1.87$ & 0.054 \\
\hline High & 1.71 & $1.27-2.31$ & $<0.001$ & 1.43 & $1.05-1.95$ & 0.025 \\
\hline \multicolumn{7}{|c|}{ Suspected invasive component biopsy } \\
\hline No & 1 & & & 1 & & \\
\hline Yes & 4.32 & $3.09-6.04$ & $<0.001$ & 3.84 & $2.69-5.46$ & $<0.001$ \\
\hline
\end{tabular}

${ }^{\mathrm{a}}$ For all interaction variables $p>0.05$ : grade and suspect, $p=0.469$, age $<45$ years; palpable, $p=0.168$, age $<45$ years; BI-RADS, $p=0.996$, age $<45$ years; DCIS grade, $p=0.108$

based on the imputed dataset

'Age: continuous: $p=0.552$, quadratic relationship (adding a quadratic term): $p=0.257$, dichotomous with threshold 40 years: $p=0.923$, dichotomous with threshold 45 years: $p=0.421$, dichotomous with threshold 55 years: $p=0.644$

underestimation was on average $27.4 \%$ for the biopsy of DCIS that were underestimated invasive breast cancers, whereas it was on average $18.8 \%$ for the biopsy of DCIS that also had the DCIS diagnosis at excision. The predicted risks for each combination of risk factors are shown in supplement 2 . The matching of the predicted risks with the observed rate is shown in supplement 3 .

The ability of the model to separate DCIS as diagnosis after excision from underestimated invasive breast cancer is shown in Fig. 1. To draw this figure, the DCIS were divided into low-risk or high-risk DCIS based on a cut-off point, and for each point the sensitivity and 1-specificty was calculated. In this study, the sensitivity means the rate of underestimated invasive breast cancer that was correctly predicted as high risk, and 1-specificity means the rate of DCIS at excision that was falsely predicted as high risk. The AUC (c-index) of the ROC was 0.668 and the AUC, corrected for optimism by bootstrapping, was 0.661 . The AUC for a model based only on lesions $>5 \mathrm{~mm}$ was 0.69 .

Based on the predicted risks, the DCIS biopsies were divided into five subgroups; the characteristics of each subgroup are shown in Table 4. From the subgroups with the lowest predicted risk to the subgroup with the highest predicted risk, the underestimation rate increased from 10.7 to $40.1 \%$.

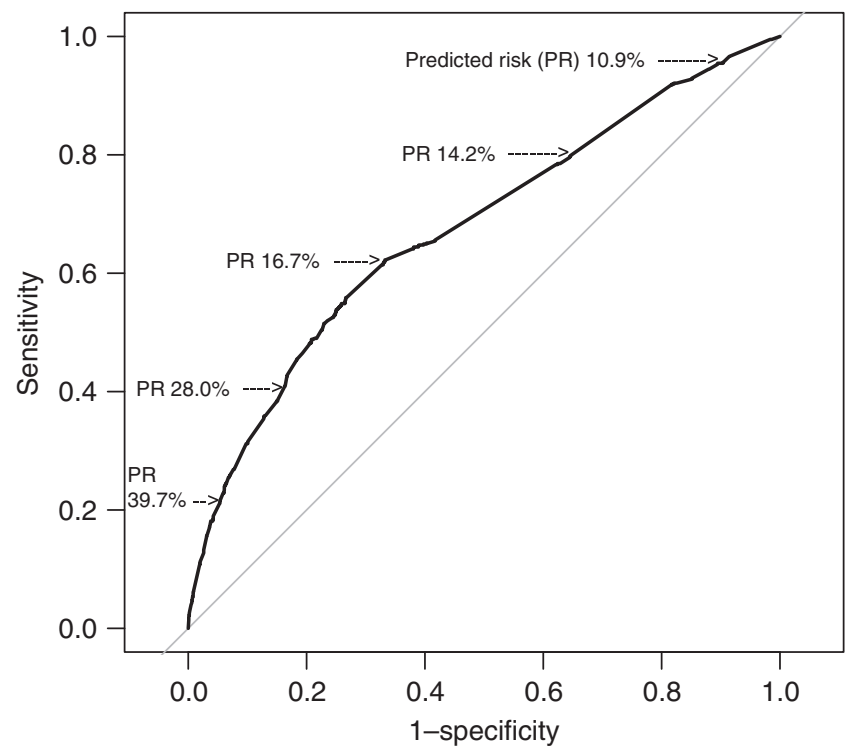

Fig. 1 Performance of the model in relation to the chosen cut-off point of the predicted risks

The associations between the predicted risks and minimalvolume DCIS were as follows: the rates of minimal-volume DCIS decreased from 18.0 to $1.6 \%$ from the subgroups with the lowest predicted risk to the subgroup with the highest predicted risk, $p<$ 0.001 (see Table 4). On average, $6.8 \%$ of DCIS diagnoses at biopsy were minimal-volume DCIS, in which the DCIS was completely removed via biopsy (meaning $8.5 \%$ of the 2296 lesions with the DCIS diagnosis at excision).

The associations between the predicted risks and unfavourable features were as follows: the percentage of invasive breast cancers with unfavourable features increased from 15.9 to $49.5 \%$ from the lowest to the highest predicted risk group, $p<0.001$ (see Table 4). On average, $39 \%$ of the invasive breast cancers had unfavourable features. More details on the distribution of tumour characteristics of the 596 invasive breast cancers are shown in supplement 4 . Of the invasive breast cancers, $27 \%$ were grade III, 26\% were Her2Neu positive, $8 \%$ were triple negative, $77 \%$ were TNM stage $1 \mathrm{~A}$ (size at maximum $2.0 \mathrm{~cm}$ and no metastasis) and the median size was $6 \mathrm{~mm}$.

\section{DISCUSSION}

The aim of our study was to expand the knowledge on underestimation of invasive breast cancer at core-needle biopsy in the routine clinical practice in the Netherlands and to develop a prediction model based on the analysis of a retrospective population-based dataset of 2892 DCIS diagnoses. We also analysed the association of predicted risk with minimal-volume DCIS and with the occurrence of unfavourable features of the underestimated invasive breast cancer.

The risk for underestimation of invasive breast cancer after a DCIS diagnosis was almost $21 \%$. Preoperatively known risk factors for an underestimated diagnosis of invasive breast cancer were a high DCIS grade, a palpable tumour, a BI-RADS score 5 and a histologically suspected invasive component. Detection mode was also included in the model, although the association with underestimation was comparably weak. The predicted risk for underestimation ranged from 9.5 to $80.2 \%$. Of the 596 underestimated invasive breast cancers, $39 \%$ had unfavourable features. Of the DCIS diagnoses at excisional pathology, $6.8 \%$ were minimal-volume DCIS. 
The underestimation rate of $20.6 \%$ shows that excision of the DCIS is still not only important for preventing DCIS from progressing to invasive breast cancer but also for finding already existing invasive breast cancers. The rate found in our study was in between the $25.9 \%$ of a meta-analysis published in 2011 and the recently reported $14.1 \%$ of a large single institution study. ${ }^{14,16}$ The underestimation rate is associated with the diagnostic work-up whereby there is a tendency to decreasing underestimation rates in more recent time period. This study used data from 2011 and 2012. At that time vacuum-assisted biopsy was not yet commonly used in the Netherlands, therefore we assume that the underestimation rate currently will be somewhat lower in the Netherlands. And in the period 2011-2012, hospitals often used screen film mammography, but the screening mammography was already digitized, and therefore no major difference in underestimation rate. The Netherlands currently is assumed because of this change in technique.

This population-based study showed several clinical, radiological and pathological features that are all routinely available before operation as risk factors for underestimation.

The risk factors we found are partly similar to those reported in literature. Differences could be due to sample size, as this study was much larger than other studies: studies in literature had 172 to 834 cases and up to 145 events, whereas we had 2892 cases and 589 events. Differences in study outcomes could also be caused by the combination of available data and the correlation between many data. For age, others found various risks for the youngest age category: no increase ${ }^{25}$, increased but not significantly so $^{16}$ and univariable significant but not in multivariable analysis ${ }^{14,21}$. In our study, young age was also only univariably associated with underestimation. For DCIS grade, the risk of underestimation for intermediate grade was in between the risk for low-grade and high-grade DCIS. This was also reported by some other studies ${ }^{14,20,27}$, whereas others reported the risk for intermediate-grade DCIS as comparable to that of the high-grade risk $^{19,25}$. In our study the DCIS grade was less discriminative than the other risk factors in the model, but on the other hand the underestimation rate of $15 \%$ for low DCIS grade was the lowest rate for a subgroup in the model and high grade was the largest subgroup with an increased risk. Palpability of the lesion has consistently been reported as a risk factor, which this study could confirm. ${ }^{15,16,18,19,22-24,26,37}$ The BI-RADS score is an assessment categorization that should give an indication of the likelihood of cancer based on the interpretation of the radiologist. We showed that it is associated with the underestimation rate; the difference between BI-RADS score 4 and 5 was 23\% in underestimation rate, which is much larger than the $7-8 \%$ found by others. ${ }^{16,21}$ A larger difference was reported in a study with a high average underestimation rate due to a high rate of micro-invasion. ${ }^{15}$ Still, the study of Kim et al. $^{15}$ is interesting because they found a somewhat higher underestimation rate for BI-RADS score $4 \mathrm{C}$, compared to $4 \mathrm{a}$ and $4 \mathrm{~b}$. It is worth noting that the BI-RADS score has not yet been investigated very extensively. A suspected invasive component has also only been reported in a limited number of studies. ${ }^{23,24}$; all found a high risk for underestimation for biopsies with a suspected component.

The prediction model we developed with the identified risk factors must be used wisely. For selecting high-risk lesions, it has to be noted that lesions with a high predicted risk still have a good chance of a final diagnosis of DCIS since the sensitivity of the model was low. The sensitivity or the AUC was higher in several other studies. ${ }^{14,17,22,24,28}$ Each study with a prediction model used different risk factors and therefore the models are not easily comparable. This has also been demonstrated in external validation of studies that applied published models to their cases; one study demonstrated a tendency towards lower or higher numbers of observed underestimates than expected ${ }^{29}$, and another previous study demonstrated validation AUCs of 
0.59-0.66, whereas the studies they validated reported validities of $0.70-0.85^{14}$. The low AUC in this study could also be due to the absence of certain data that might have been important, such as the type of biopsy device and the size of the lesion on mammography. This is shown in Table 1, where the references that were made bold are the results of the studies making a prediction model, whereas the variable names that are given in bold are the variables that were analysed in this study.

Part of the DCIS was minimal-volume DCIS and was thus removed from the biopsy itself. In this study, minimal-volume DCIS was associated with the predicted underestimation risk. To our knowledge, this information has never been demonstrated before; one study demonstrated a similar rate of minimal-volume DCIS, but the association with underestimation was not investigated. ${ }^{31}$ In our study, the minimal-volume DCIS was higher for the predicted low-risk group.

The invasive breast tumours that were found at excision were heterogeneous in prognostic and predictive features. Underestimated invasive tumours are often small: the median size was 6 $\mathrm{mm}$, which is in line with or somewhat lower than the results of other studies. ${ }^{17,25-27}$ On the other hand, $8 \%$ were TNM stage IIB or III, and $20 \%$ were triple negative or ER-PR-Her2Neu+. Where other studies analysed none or a few tumour characteristics, we had numerous tumour-related data of the 589 underestimates. Based on these data, we calculated the rate of cancers with unfavourable features, which was $39 \%$. For these patients, systemic therapy was indicated. In our study, the rate of unfavourable features was higher for the predicted high-risk DCIS group.

Due to its retrospective nature, this study has certain limitations. A limitation in interpreting the results is that the preoperative decisions and techniques were not standardized, and therefore the preferences of the treating physicians and the patients will have influenced the underestimation results. For instance, for a high-grade DCIS with histological suspicion of invasiveness, the biopsy can be repeated (and invasive breast cancer might be found preoperatively) or initial treatment can be started (with an increased risk of underestimation). Also, for DCIS grade, other studies might have used different grading systems. Another limitation is that results of observational studies are difficult to compare because of differences in diagnostic work-up, differences in major selection criteria, such as the presence of micro-invasion, differences in investigated risk factors and associations between the investigated risk factors. Our dataset did not provide information on the number of biopsies nor on the biopsy device, and hence the amount of tumour taken at biopsy was not known. Some other factors were not available either, such as the presence of comedo-necrosis, the breast density, the visibility of the lesion on ultrasound, the presence of mammographic mass or the size of the lesion seen on the mammogram.

The model in this study is based on a large dataset that is based on nationwide Dutch data, and it demonstrated the association of risk for underestimation with minimal-volume DCIS and unfavourable features of invasive cancer, which makes the results valuable. The prediction model could be improved by adding additional data; the most interesting targets of investigation for future research are the biopsy type and mammography-related data: $\mathrm{BI}-$ RADS score 4 subcategories, the underlying reasons for a BI-RADS score (such as mass), size of the lesion and presence of residual mammographic abnormalities after biopsy. Furthermore, the prediction model should be validated externally.

\section{CONCLUSION}

Our results demonstrated that the risk for an underestimated diagnosis of invasive breast cancer after a diagnosis of DCIS at biopsy is considerable. Of these invasive breast cancers, two-fifths has unfavourable features. With our prediction model, the individual risk of underestimation can be calculated based on routinely available preoperatively known risk factors

\section{ACKNOWLEDGEMENTS}

The authors thank the Netherlands Comprehensive Cancer Organization and the PALGA foundation for providing a registration database and for their efforts in making the research database for this study.

\section{AUTHOR CONTRIBUTIONS}

Study conception: C.J.C.M/P.J.W., study design: C.J.C.M./J.v.R./M.B.E.M.-P./P.J.W., data coding: C.J.C.M./P.J.W., data analyses: C.J.C.M./J.v.R., data interpretation: C.J.C.M./J.v.R./ M.B.E.M.-P./B.A.M.t.B./L.d.M./S.S./P.J.W., drafting article: C.J.C.M., revision article: J.v.R./ M.B.E.M.-P./B.A.M.t.B./L.d.M./S.S./P.J.W.

\section{ADDITIONAL INFORMATION}

Supplementary information is available for this paper at https://doi.org/10.1038/ s41416-018-0276-6.

Competing interests: The authors declare no competing interests.

Ethics approval: The study was approved by the scientific committee of PALGA (14.025 LZV1073) and the Privacy Review Board of IKNL (K14.021).

Data availability: The dataset generated for this current study are not publicly available due additional research questions to be answered, but is available from the corresponding author on reasonable request. The prediction model is available for external validation via Evidencio (model 1074).

Funding: This work was supported by the Dutch Cancer Foundation (KWF), grant SLP2015-7769.

Note: This work is published under the standard license to publish agreement. After 12 months the work will become freely available and the license terms will switch to a Creative Commons Attribution 4.0 International (CC BY 4.0).

\section{REFERENCES}

1. Nationaal Borstkanker Overleg Nederland. Dutch Breast Cancer Guideline, DCIS [Internet]. 2012 [cited 1 Jun 2017]. p. version 2. Available from: https:// richtlijnendatabase.nl/en/richtlijn/breast_cancer/locoregional_treatment/dcis/ treatment of dcis.html

2. NICE. Clinical Guideline 80; Early and Locally Advanced Breast Cancer: Diagnosis and Treatment [Internet]. 2017 [cited 1 Jun 2017]. https://www.nice.org.uk/guidance/ CG80/chapter/1-Guidance\#referral-diagnosis-and-preoperative-assessment

3. Van Luijt, P. A. et al. The distribution of ductal carcinoma in situ (DCIS) grade in 4232 women and its impact on overdiagnosis in breast cancer screening. Breast Cancer Res. 18, 47 (2016).

4. Erbas, B., Provenzano, E., Armes, J. \& Gertig, D. The natural history of ductal carcinoma in situ of the breast: a review. Breast Cancer Res. Treat. 97, 135-144 (2006).

5. Sanders, M. E., Schuyler, P. A., Simpson, J. F., Page, D. L. \& Dupont, W. D. Continued observation of the natural history of low-grade ductal carcinoma in situ reaffirms proclivity for local recurrence even after more than 30 years of follow up. Mod. Pathol. 28, 662-669 (2015).

6. Elshof, L. E. et al. Feasibility of a prospective, randomised, open-label, international multicentre, phase III, non-inferiority trial to assess the safety of active surveillance for low risk ductal carcinoma in situ-The LORD study. Eur. J. Cancer 51, 1497-1510 (2015)

7. Wesseling, J., Peric, A. \& Tryfonidis, K. Management of Low-Risk DCIS (LORD) [Internet]. ClinicalTrials.gov. 2015 [cited 12 Apr 2017]. https://clinicaltrials.gov/ show/NCT02492607

8. Francis, A. et al. Addressing overtreatment of screen detected DCIS: the LORIS Trial. Eur. J. Cancer 51, 2296-2303 (2015).

9. Soumian, S. et al. Concordance between vacuum assisted biopsy and postoperative histology: Implications for the proposed Low Risk DCIS Trial (LORIS). Eur. J. Surg. Oncol. 39, 1337-1340 (2013).

10. Francis, A. LORIS. A Phase III Trial of Surgery versus Active Monitoring for Low Risk Ductal Carcinoma In Situ (DCIS) [Internet] (University of Birmingham, 2014). http:// www.birmingham.ac.uk/research/activity/mds/trials/crctu/trials/loris/index.aspx 
11. Hwang, S., Partridge, A. \& Thompson, A. Comparison of Operative to Monitoring and Endocrine Therapy (COMET) Trial for Low Risk DCIS [Internet]. ClinicalTrials.gov. 2016 [cited 12 Apr 2017]. https://clinicaltrials.gov/show/NCT02926911

12. Ryser, M. D. et al. Outcomes of active surveillance for ductal carcinoma in situ: a computational risk analysis. J. Natl. Cancer Inst. 108, djv372 (2016).

13. Grimm, L. J. \& Shelley Hwang, E. Active aurveillance for DCIS: the importance of selection criteria and monitoring. Ann. Surg. Oncol. https://doi.org/10.1245/ s10434-016-5596-2, 2-4 (2016).

14. Jakub, J. W. et al. A validated nomogram to predict upstaging of ductal carcinoma in situ to invasive disease. Ann. Surg. Oncol. 10, 2915-2924 (2017).

15. Kim, J. et al. Factors associated with upstaging from ductal carcinoma in situ following core needle biopsy to invasive cancer in subsequent surgical excision. Breast 21, 641-645 (2012).

16. Brennan, M. E. et al. Ductal carcinoma in situ at core-needle biopsy: meta-analysis of underestimation and predictors of invasive breast cancer. Radiology 260, 119-128 (2011).

17. Huo, L. et al. Predictors of invasion in patients with core-needle biopsy-diagnosed ductal carcinoma in situ and recommendations for a selective approach to sentinel lymph node biopsy in ductal carcinoma in situ. Cancer 107, 1760-1768 (2006).

18. Goyal, A. et al. Is there a role of sentinel lymph node biopsy in ductal carcinoma in situ?: analysis of 587 cases. Breast Cancer Res. Treat. 98, 311-314 (2006).

19. Meijnen, P. et al. Risk of invasion and axillary lymph node metastasis in ductal carcinoma in situ diagnosed by core-needle biopsy. Br. J. Surg. 94, 952-956 (2007).

20. O'Flynn, E. A. M. et al. Prediction of the presence of invasive disease from the measurement of extent of malignant microcalcification on mammography and ductal carcinoma in situ grade at core biopsy. Clin. Radiol. 64, 178-183 (2009).

21. Trentin, C. et al. Predictors of invasive breast cancer and lymph node involvement in ductal carcinoma in situ initially diagnosed by vacuum-assisted breast biopsy: experience of 733 cases. Breast 21, 635-640 (2012).

22. Lee, S. K., Yang, J. H., Woo, S. Y., Lee, J. E. \& Nam, S. J. Nomogram for predicting invasion in patients with a preoperative diagnosis of ductal carcinoma in situ of the breast. Br. J. Surg. 100, 1756-1763 (2013).

23. Park, H. S. et al. Risk predictors of underestimation and the need for sentinel node biopsy in patients diagnosed with ductal carcinoma in situ by preoperative needle biopsy. J. Surg. Oncol. 107, 388-392 (2013).
24. Coufal, O. et al. A simple model to assess the probability of invasion in ductal carcinoma in situ of the breast diagnosed by needle biopsy. Biomed. Res. Int. 2014, 480840 (2014).

25. Osako, T. et al. Incidence and prediction of invasive disease and nodal metastasis in preoperatively diagnosed ductal carcinoma in situ. Cancer Sci. 105, 576-582 (2014).

26. Sato, Y. et al. Preoperatively diagnosed ductal carcinoma in situ: risk prediction of invasion and effects on axillary management. Breast Cancer 23, 761-770 (2015).

27. Caswell-Smith, P. \& Wall, M. Ductal carcinoma in situ: Is core needle biopsy ever enough? J. Med. Imaging Radiat. Oncol. 61, 29-33 (2017).

28. Park, H. S. et al. A nomogram for predicting underestimation of invasiveness in ductal carcinoma in situ diagnosed by preoperative needle biopsy. Breast 22, 869-873 (2013).

29. Lee, S. C. \& Chang, M. C. Development and validation of web-based nomograms to predict postoperative invasive component in ductal carcinoma in situ at needle breast biopsy. Healthc. Inform. Res. 20, 152-156 (2014).

30. Kondo, T. et al. A model to predict upstaging to invasive carcinoma in patients preoperatively diagnosed with ductal carcinoma in situ of the breast. J. Surg. Oncol. [Internet] 112, 476-480 (2015).

31. Muhsen, $\mathrm{S}$. et al. Outcomes for women with minimal-volume ductal carcinoma in situ completely excised at core biopsy. Ann. Surg. Oncol. 24, 3888 (2017).

32. Casparie, M. et al. Pathology databanking and biobanking in The Netherlands, a central role for PALGA, the nationwide histopathology and cytopathology data network and archive. Cell. Oncol. 29, 19-24 (2007).

33. Van der Sanden, G. A. C., Coebergh, J. W. W., Schouten, L. J., Visser, O. \& Van Leeuwen, F. E. Cancer incidence in the Netherlands in 1989 and 1990: first results of the nationwide Netherlands cancer registry. Eur. J. Cancer 31, 1822-1829 (1995).

34. Zonderland, H. M. BI-RADS classification and breast cancer. Application in the Netherlands. Ned. Tijdschr. Oncol. 6, 145-157 (2009).

35. Brierley, J. D., Gospodarowicz, M. K. \& Wittekind, C. TNM Classification of Malignant Tumours 7th edn, John Willey \& Sons, West Sussex, UK, 181-193 (2009).

36. Nationaal Borstkanker Overleg Nederland. Dutch Breast Cancer Guideline, Invasive Breast Cancer [Internet]. 2012 [cited 2017 Jul 7]. p. version 2. https:// richtlijnendatabase.nl/en/richtlijn/breast_cancer/adjuvant_systemic_therapy. html\#onderbouwing

37. Van Zee, K. J. Use of axillary staging in the management of ductal carcinoma in situ. JAMA Oncol. 1, 332 (2015). 